\title{
Genetic aspects of antibiotic induced deafness: mitochondrial inheritance
}

\author{
Dan-Ning Hu, Wei-Qin Qiu, Bao-Tong Wu, Li-Zhou Fang, Fu Zhou, Yue-Ping Gu, \\ Qiu-Hua Zhang, Jian-Hua Yan, Yu-Qin Ding, Hong Wong
}

\begin{abstract}
Analysis of 36 pedigrees with a positive family history of aminoglycoside antibiotic induced deafness, ascertained in a population of 483611 in Zhabei District in Shanghai, showed that the susceptibility to antibiotic ototoxicity was transmitted by females exclusively, indicating mitochondrial inheritance. Reanalysis of 18 other published pedigrees confirmed this conclusion.
\end{abstract}

Aminoglycoside antibiotics(streptomycin, kanamycin, gentamicin, tobramycin, neomycin) can lead to hearing loss. The incidence of aminoglycoside antibiotic induced deafness (AAID) has increased over recent years and is a major cause of deafness in China. ${ }^{12}$ The hearing loss is toxic in nature, but marked individual difference in the response to the ototoxic effect of antibiotics has been reported. ${ }^{3}$ Familial aggregation of AAID has been reported. ${ }^{4-14}$ Some patients developed AAID after treatment with conventional doses over a short period, indicating that some subjects may have a genetically determined susceptibility to the antibiotics. So far, only isolated pedigrees have been reported and no large survey on this subject has been published. The genetic aspects of susceptibility to antibiotic ototoxicity have not been completely elucidated, although some authors have

Department of Genetic Counselling, National Centre of Genetic Medicine, and Department of Medical Genetics, Tiedao Medical College, Shanghai, China.

D-N Hu, W-Q Qiu

Department of Population Genetics, Zhabei Eye Hospital, Shanghai, China.

D-N Hu, F Zhou, Q-H Zhang, J-H Yan, Y-Q Ding, H Wong

Department of Oto-Rhino-Laryngology, Zhabei Central Hospital, Shanghai, China.

B-T Wu, L-Z Fang, Y-P Gu

Correspondence to Dr D-N Hu, Block 2, 19/F Flat C, Hoi Kwong Court, Hoi Kwong Street, Hong Kong.

Received for publication 23 February 1990.

Revised version accepted for publication 9 July 1990. suggested autosomal dominant inheritance. ${ }^{69}$ This paper is concerned with the mode of inheritance of susceptibility to antibiotic ototoxicity, based on complete ascertainment of AAID in a district in Shanghai with a population of 483611 , and investigation of 36 pedigrees of AAID cases found during this study.

\section{Subjects and methods}

Zhabei district is one of 12 districts in Shanghai City. A list of deaf mutes was obtained from the Zhabei Association of Deaf Mutism, and supplemented by the area's welfare officers, two schools for deaf children, and three factories for deaf mutes in Zhabei District. Every case of deaf mutism is registered with the Zhabei Association of Deaf Mutism and are known to their area's welfare officers; most of them are either in schools for deaf children or work in factories for deaf mutes. All these subjects were contacted, so that ascertainment could be assumed to be reasonably complete. Children under 7 years old were excluded from this study, since the diagnosis is uncertain before that age.

Every deaf mute discovered in this study was visited by two of the authors. A detailed history regarding the age of onset and knowledge of pathogenesis (infection, drug, etc) was obtained. Each case was examined by an otologist and audiometry was performed when necessary. In cases of AAID, a detailed family history was obtained for each proband, including the occurrence of AAID in all relatives (first, second, and third degree). The history of the use of antibiotics in every AAID case was investigated in detail, including the drug, duration of therapy, the disease needing antibiotic treatment, and the age of onset of AAID.

The severity of hearing loss was classified as follows. (1) Total or near-total deafness: hearing loss $<90 \mathrm{~dB}$, occurring in early childhood, which can lead to deaf mutism. (2) Severe deafness: hearing loss 70 to $90 \mathrm{~dB}$, with difficulty in hearing normal speech and only able to hear loudly spoken words. (3) Mild to moderate deafness: hearing loss 27 to $70 \mathrm{~dB}$ with little or partial difficulty in hearing normal speech. 


\section{Results}

PREVALENCE

A total of 763 deaf mutes was found in a population of 483611 in Zhabei district. Two hundred and eightyfive cases were diagnosed as congenital deafness, there was one case of $X$ linked recessive deaf mutism with late onset, and seven cases of genetic deafness syndromes (Usher's syndrome, Waardenburg's syndrome, Franceschetti's syndrome, and deafness associated with atresia of the external auditory meatus): Four hundred and seventy cases were diagnosed as acquired deaf mutism, as hearing loss occurred in early infancy and a definite aetiological factor could be found in the history (such as infection, drugs, trauma, etc), including 168 cases caused by drugs. Among the drug induced deaf mutes, only one case was caused by quinine and the other 167 cases were caused by aminoglycoside antibiotics. The prevalence of AAID was $0.035 \%$ (1:2896), accounting for $21.9 \%$ of all deaf mutes.

\section{AAID WITH POSITIVE FAMILY HISTORY}

In 167 AAID cases, 47 cases had at least one family member (including first, second, and third degree) who suffered from AAID, accounting for $28 \cdot 1 \%$ of all AAID cases and $6 \cdot 2 \%$ of all deaf mutes. The prevalence of AAID with a positive family history was $0 \cdot 0097 \%(1: 10290)$. Some of the cases belonged to the same family, so there were 36 pedigrees altogether. A detailed investigation was undertaken in these 36 pedigrees, during which another 61 cases of AAID were discovered. Taken with the 47 probands, there were 108 cases of AAID in 36 pedigrees.

Sex

Of 108 cases, 58 were male and 50 cases were female.

\section{Age of onset}

Definite age of onset could be ascertained in 90 of the 108 AAID cases (table 1). Most patients had onset before 7 years of age and half the cases occurred before 3 years of age.

\section{Diseases treated with antibiotics}

Of the 108 cases, 90 cases could remember the

Table 1 Age of onset in AAID with a positive family history.

\begin{tabular}{lrr}
\hline Age of onset & No of cases & $\%$ \\
\hline $0-$ & 23 & $25 \cdot 6$ \\
$1-$ & 22 & 24.4 \\
$3-$ & 29 & $32 \cdot 2$ \\
$7-$ & 8 & 8.9 \\
$17-$ & 8 & 8.9 \\
Total & 90 & $100 \cdot 0$ \\
\hline
\end{tabular}

Table 2 Diseases needing antibiotic therapy in AAID with a positive family history.

\begin{tabular}{lc}
\hline Disease & No of cases \\
\hline Upper respiratory infection & 48 \\
Bronchitis & 9 \\
Measles & 6 \\
Pneumonia & 4 \\
Gastroenteritis & 4 \\
Meningitis & 3 \\
Tonsillitis & 2 \\
Pulmonary tuberculosis & 1 \\
Others & 12 \\
Total & 89 \\
\hline
\end{tabular}

Table 3 Duration of therapy in AAID cases.

\begin{tabular}{lcc}
\hline $\begin{array}{l}\text { Duration of } \\
\text { therapy (days) }\end{array}$ & $\begin{array}{c}\text { Case with } \\
\text { positive family } \\
\text { history }\end{array}$ & $\begin{array}{c}\text { Cases without } \\
\text { positive family } \\
\text { history }\end{array}$ \\
\hline $1-7$ & $46(59 \cdot 0 \%)$ & $2(10 \cdot 0 \%)$ \\
$8-30$ & $24(30 \cdot 8 \%)$ & $9(45 \cdot 0 \%)$ \\
$>30$ & $8(10 \cdot 2 \%)$ & $9(45 \cdot 0 \%)$ \\
Total & $78(100 \%)$ & $20(100 \%)$ \\
\hline
\end{tabular}

diseases that needed antibiotic treatment. Table 2 shows that most of the patients suffered from nonspecific infections, such as upper respiratory infections, bronchitis, tonsillitis, gastroenteritis, etc. Only one case suffered from pulmonary tuberculosis.

\section{Antibiotics}

Of the 108 cases, the antibiotics used could be ascertained in 90 cases, and included 80 cases of streptomycin, two cases of kanamycin, and eight cases of a combination of two or three antibiotics (streptomycin, kanamycin, gentamicin).

\section{Dosage and duration of therapy}

Most cases were treated with a conventional daily dosage, except one case treated with a double daily dosage. In order to investigate whether AAID cases with a positive family history have a high susceptibility to antibiotic ototoxicity, we compared the duration of therapy of the present series with a control group of 20 cases of AAID without a positive family history (table 3). Table 3 shows that the duration of therapy was definitely shorter in the group with a positive family history, indicating that high susceptibility existed in this group. Of 78 cases with a positive family history, the duration of therapy in 70 cases $(89.8 \%)$ was 30 days or less and in 46 cases $(59.0 \%)$ was seven days or less, with an average of 14.7 days. In two cases hearing loss occurred after one day of treatment with streptomycin. In the control group, the duration of therapy was more than seven days in 


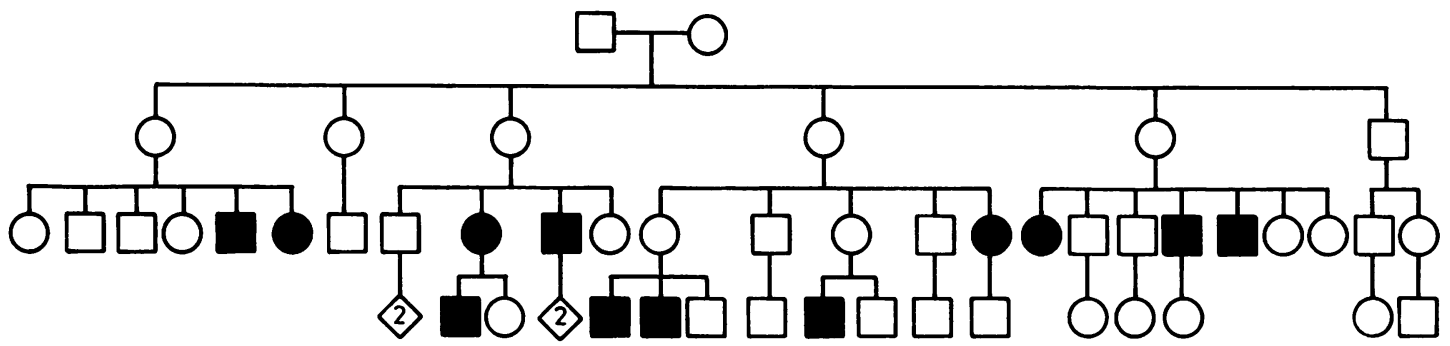

Figure 1 Pedigree 8(present series).

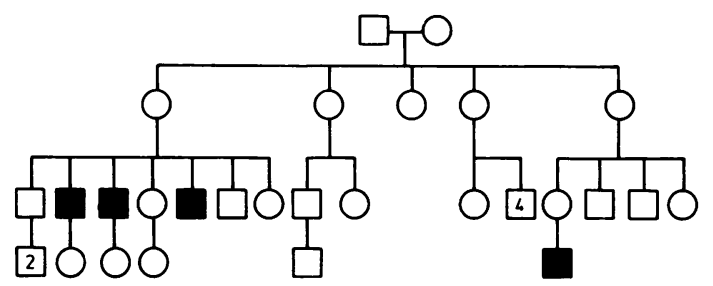

Figure 2 Pedigree 12 (present series).

18 cases $(90.0 \%)$, with an average of 89.0 days. The difference between the two groups was statistically significant $\left(\chi^{2}\right.$ test, $\left.p>0.01\right)$.

\section{Severity of deafness}

Of the 108 cases, the severity of deafness could be ascertained in 90 cases, with 57 cases of total or neartotal deafness, 31 cases of severe deafness, and two cases of mild to moderate deafness.

\section{ANALYSIS OF PEDIGREES}

Thirty-six pedigrees in the present series can be divided into two groups.

(1) Transmission could be ascertained. In 22 pedigrees, transmission could be ascertained, including nine pedigrees with direct vertical transmission (both mother and children affected) (pedigrees 1 to 9) (fig 1). There were 13 pedigrees without direct transmission, but with several members affected (such as cousins); these could be traced to a common ancestor, so the carriers could be ascertained (pedigrees 10 to 22) (fig 2). All of the 22 pedigrees had in common that the transmission, whether directly or through carriers, was exclusively through females. Affected males did not transmit the characteristic to their offspring (there was one AAID case where both parents were affected), indicating that the inheritance in our series was through the maternal line.

(2) Transmission could not be ascertained. In 14 pedigrees, only the sibs of the probands were affected, so the carrier could not be ascertained.

\section{Discussion}

The incidence of AAID increased rapidly after the introduction of widespread use of antibiotics. In the 1950 s and 1960s, AAID cases accounted for 0.36 to $1.20 \%$ of all deaf mutes in China and increased to 12.8 to $66.1 \%$ of all deaf mutes in the $1980 \mathrm{~s},{ }^{12}$ being one of the most important causes of deaf mutism.

Familial aggregation of AAID has been reported; Sheng ${ }^{14}$ reported that cases with a positive family

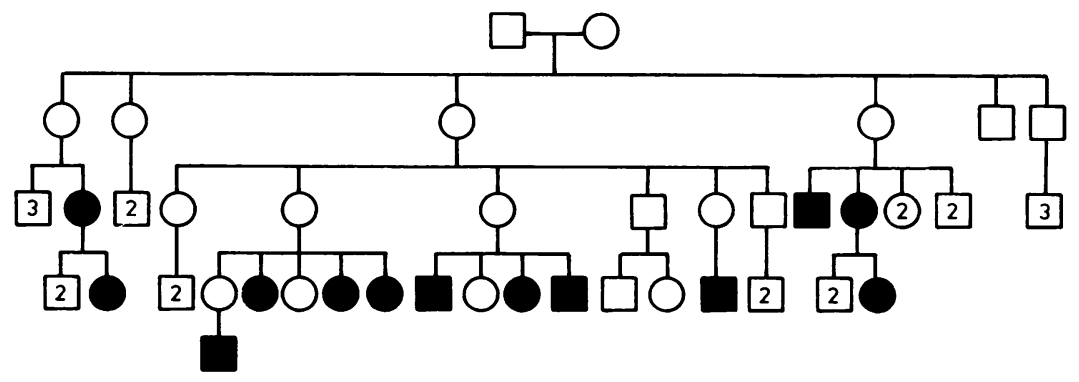

Figure 3 Pedigree 32 (reported by Sun et al ${ }^{12}$ ). 
history accounted for $14 \cdot 3 \%$ and $18 \cdot 0 \%$ of gentamicin induced deafness and streptomycin induced deafness, respectively, ${ }^{14}$ as compared with $28 \cdot 1 \%$ in the present series.

Two possible interpretations exist for the familial aggregation of AAID. One is that susceptibility to antibiotic ototoxicity exists in some families and the predisposition is inherited. Another interpretation is that as some contagious diseases (such as tuberculosis) can occur in several members of a family and need long term antibiotic treatment, the basis for familial aggregation of AAID is environmental rather than hereditary. In the present series, most cases of AAID were treated for acute, non-specific infections rather than chronic contagious diseases such as tuberculosis, different members of the same family were usually treated for different diseases at different times, and the duration of therapy was definitely shorter in the cases with a positive family history, indicating that the familial aggregation of AAID could be better explained on a genetic basis.

To our knowledge, 18 pedigrees of familial aggregation of AAID have been published. ${ }^{4-13}$ Some authors have reported autosomal dominant inheritance. ${ }^{6} 9$ Transmission could be traced in 15 pedigrees, including 33 patients with affected mothers. All of the fathers were unaffected, except in one family where both the father and mother were affected. In four patients, both parents were unaffected, but could be traced to an affected female ancestor through the female carriers. In 24 patients, no direct transmission could be traced, but other members of the family were affected (such as cousins), and could be traced to a common ancestor through the female carriers (pedigrees 23 to 37, fig 3). Reanalysis of the 15 pedigrees showed exclusively female transmission, confirming that the susceptibility to antibiotic ototoxicity is inherited through the maternal line. The possibility of $\mathrm{X}$ linked inheritance should also be considered. However, both sexes were equally affected and affected males did not transmit the susceptibility to their daughters, nor to their grandsons through an unaffected daughter. So $\mathrm{X}$ linked dominant or recessive inheritance can be excluded.

Maternal transmission can be explained by mitochondrial inheritance ('cytoplasmic inheritance' in the old terminology). Mitochondrial DNA has 16569 base pairs, equivalent to 5523 codons. Most of the mtDNA serves a coding function and contains no intervening sequences and little in the way of flanking sequences. 1516

The mtDNA is transmitted exclusively by women. Spermatozoa, which contain hardly any cytoplasm, cannot transmit mtDNA. Human disorders which have been explained by mitochondrial inheritance include Leber's hereditary optic neuropathy, KearnsSayre syndrome, and infantile bilateral striated necrosis. ${ }^{15}$ 17-20
Analysis of the sequence of mtDNA indicated that a simple nucleotide change of position 11778 was associated with Leber's hereditary optic neuropathy. ${ }^{19}$ Deletion of muscle mtDNA was found in patients with Kearns-Sayre syndrome. ${ }^{20}$ Chloramphenicol resistance in cultured human cells is determined by the mitochondrial genome. Enucleated resistant cells transmit the character to sensitive cells when fused with them. The precise nucleotide changes in $16 \mathrm{~S}$ rRNA are known. ${ }^{21} 22$ A dose related toxic effect of chloramphenicol occurs presumably in all persons who take enough. An idiosyncratic reaction, which is genetically determined, occurs in about 1 in every 19000 persons taking the drug. Of three reported instances of familial chloramphenicol toxicity, two involved identical twins and the other involved a man and his sister's daughter, consistent with mitochondrial inheritance. ${ }^{23} 24$ Given that mitochondrial inheritance is the mode of inheritance of susceptibility to antibiotic ototoxicity, aminoglycoside antibiotic therapy should be avoided in those persons with a family history of ototoxic side effects from the drug (especially the maternal relatives).

Only a few human disorders have been shown to be mitochondrially inherited, all being rare diseases. Susceptibility to antibiotic ototoxicity is quite common, the prevalence of AAID with a positive family history being about 1:10 000 in the present series. Many people have never taken aminoglycoside antibiotics, so the actual prevalence of persons with susceptibility to antibiotic ototoxicity should be much higher. This would provide a wide field for the study of mitochondrial inheritance in man.

1 Fu DM. Survey of 1583 deaf mutes. Qinghai Med f 1985;1:22-3.

$2 \mathrm{Lu} \mathrm{YF}$. Cause of 611 deaf mutes in schools for deaf children in Shanghai. Shanghai Med 1987;10:159.

3 Prazic M, Salaj B. Ototoxicity with children caused by streptomycin. Audiology 1975;14:173-6.

4 Horiguchi I. Familial streptomycin-induced hearing loss. fpn $\mathcal{f}$ Otorhinolaryngol 1957;29:1396.

5 Prazic M, Salaj B, Subotic R. Familial sensitivity to streptomycin. f Laryngol Otol 1964;78:1037-43.

6 Tsuiki T, Murai S. Familial incidence of streptomycin hearing loss and hereditary weakness of the cochlea. Audiology 1971;10: 315-22.

7 Johnsonbaugh RE, Drexel HG, Light IJ, Southerland JM. Familial occurrence of drug-induced hearing loss. Am $\mathcal{F}$ Dis Child 1974;127:245-7.

8 Donald RR, Sellars SL. Streptomycin ototoxicity in the unborn child. South Med f 1981;66:316-8.

9 Vijoen DL, Sellars SL, Beighton P. Familial aggregation of streptomycin ototoxicity: autosomal dominant inheritance. f Med Genet 1983;20:357-60.

10 Hou XP. Familial streptomycin-induced ototoxicity: report of four cases. Adv Med 1978;9:185-6.

11 Yan FC. Familial streptomycin ototoxicity: report of one pedigree with nine cases. Chin $\mathcal{F}$ Otorhinolaryngol 1979;14:27-8.

12 Sun ZH, Zhang LY, Zhou YF. Familial streptomycin ototoxicity. Chin f Otorhinolaryngol 1986;21:143-4.

13 Sheng JH. Antibiotic toxic deafness can be inherited? Popular Med 1986;11:7-8.

14 Sheng JH. Gentamicin-induced deafness in childhood: report of 175 cases. Chin 7 Otorhinolaryngol 1985;20:135-7.

15 McKusick V. Mendelian inheritance in man. 8th ed. Baltimore: Johns Hopkins University Press, 1988. 
16 Anderson S, Bankier AT, Barrell BG, et al. Sequence and organization of the human mitochondrial genome. Nature 1981;290:457-64.

17 Eggers J, Wilson J. Mitochondrial inheritance in a mitochondrially mediated disease. $N$ Engl.7 Med 1983;309:142-6.

18 Fine PEM. Mitochondrial inheritance and disease. Lancet 1978;i: $659-62$.

19 Wallace DC, Singh G, Lott MT, et al. Mitochondrial DNA mutation associated with Leber's hereditary optic neuropathy. Science 1988;242:1427-30.

20 Zeviani M, Moraes CT, DiMauro S, et al. Deletion of mito- chondrial DNA in Kearns-Sayre syndrome. Neurology 1988;38: $1339-46$.

21 Kearsey SS, Craig IW. Altered ribosomal RNA genes in mitochondria from mammalian cells with chloramphenicol resistance. Nature 1981;290:607-8.

22 Mitchel $\mathrm{CH}$, Attardi G. Cytoplasmic transfer of chloramphenicol resistance in a human cell line. Somatic Cell Genet 1978;4: 737-44.

23 Nago T, Mauer AM. Concordance for drug-induced aplastic anemia in identical twins. $N$ Engl $\mathcal{F}$ Med 1969;281:7-11.

24 Rosenthal RL, Blackman A. Bone-marrow hypoplasia following use of chloramphenicol eye drops. FAMA 1965;191:136-7. 